\title{
On the Quadratic Irrationals, Quadratic Ideals and Indefinite Quadratic Forms
}

\author{
AHMET TEKCAN AND HACER ÖZDEN
}

\begin{abstract}
Let $P$ and $Q$ be two rational integers, $D \neq 1$ be a positive non-square integer, and let $\delta=\sqrt{D}$ or $\frac{1+\sqrt{D}}{2}$ be a real quadratic irrational with trace $t=\delta+\bar{\delta}$ and norm $n=\delta \bar{\delta}$. Given any quadratic irrational $\gamma=\frac{P+\delta}{Q}$, there exist a quadratic ideal $I_{\gamma}=[Q, \delta+P]$ and an indefinite quadratic form $F_{\gamma}(x, y)=Q x^{2}-(t+2 P) x y+\left(\frac{n+t P+P^{2}}{Q}\right) y^{2}$ of discriminant $\Delta=t^{2}-4 n$ which correspond to $\gamma$. In this paper, we obtain some properties of quadratic irrationals $\gamma$, quadratic ideals $I_{\gamma}$ and indefinite quadratic forms $F_{\gamma}$.
\end{abstract}

\section{INTRODUCTION}

A real quadratic form (or just a form) $F$ is a polynomial in two variables $x, y$ of the type

$$
F=F(x, y)=a x^{2}+b x y+c y^{2}
$$

with real coefficients $a, b, c$. The discriminant of $F$ is defined by the formula $b^{2}-4 a c$ and is denoted by $\Delta$. Moreover $F$ is an integral form iff $a, b, c \in \mathbb{Z}$ and $F$ is indefinite iff $\Delta>0$.

Let $\Gamma$ be the modular group $\operatorname{PSL}(2, \mathbb{Z})$, i.e., the set of the transformations

$$
z \mapsto \frac{r z+s}{t z+u}, r, s, t, u \in \mathbb{Z}, r u-s t=1 .
$$

$\Gamma$ is generated by the transformations $T(z)=\frac{-1}{z}$ and $V(z)=z+1$. Let $U=T \cdot V$. Then $U(z)=\frac{-1}{z+1}$. Then $\Gamma$ has a representation

2000 Mathematics Subject Classification. 11E15, 11A55, 11J70.

Key words and phrases. Quadratic irrationals, quadratic ideals, indefinite quadratic forms, extended modular group. 
$\Gamma=\left\langle T, U: T^{2}=U^{3}=I\right\rangle$. Note that

$$
\Gamma=\left\{g=\left(\begin{array}{ll}
r & s \\
t & u
\end{array}\right): r, s, t, u \in \mathbb{Z} \text { and } r u-s t=1\right\} .
$$

We denote the symmetry with respect to the imaginary axis with $R$, that is $R(z)=-\bar{z}$. Then the group $\bar{\Gamma}=\Gamma \cup R \Gamma$ is generated by the transformations $R, T, U$ and has a representation $\bar{\Gamma}=$ $\left\langle R, T, U: R^{2}=T^{2}=U^{3}=I\right\rangle$, and is called the extended modular group. Similarly,

$$
\bar{\Gamma}=\left\{g=\left(\begin{array}{ll}
r & s \\
t & u
\end{array}\right): r, s, t, u \in \mathbb{Z} \text { and } r u-s t= \pm 1\right\} .
$$

There is a strong connection between the extended modular group and binary quadratic forms (for further details see [5]). Most properties of binary quadratic forms can be given by the aid of the extended modular group. The most is equivalence of forms which is given by Gauss as follows: Let $F=(a, b, c)$ be a quadratic form and let $g=\left(\begin{array}{cc}r & s \\ t & u\end{array}\right) \in \bar{\Gamma}$. Then the form $g F$ is defined by

$$
\begin{aligned}
g F(x, y)= & \left(a r^{2}+b r s+c s^{2}\right) x^{2}+(2 a r t+b r u+b t s+2 c s u) x y \\
& +\left(a t^{2}+b t u+c u^{2}\right) y^{2}
\end{aligned}
$$

This definition of $g F$ is a group action of $\bar{\Gamma}$ on the set of binary quadratic forms. Two forms $F$ and $G$ are said to be equivalent iff there exists a $g \in \bar{\Gamma}$ such that $g F=G$. If $\operatorname{det} g=1$, then $F$ and $G$ are called properly equivalent. If $\operatorname{det} g=-1$, then $F$ and $G$ are called improperly equivalent. A quadratic form $F$ is said to be ambiguous if it is improperly equivalent to itself.

An indefinite quadratic form $F$ of discriminant $\Delta$ is said to be reduced if

$$
|\sqrt{\Delta}-2| a||<b<\sqrt{\Delta}
$$

Mollin considers the arithmetic of ideals in his book (see [1]). Let $D \neq 1$ be a square free integer and let $\Delta=\frac{4 D}{r^{2}}$, where

$$
r= \begin{cases}2 & D \equiv 1(\bmod 4) \\ 1 & \text { otherwise }\end{cases}
$$

If we set $\mathbb{K}=\mathbb{Q}(\sqrt{D})$, then $\mathbb{K}$ is called a quadratic number field of discriminant $\Delta=\frac{4 D}{r^{2}}$. A complex number is an algebraic integer 
if it is the root of a monic polynomial with coefficients in $\mathbb{Z}$. The set of all algebraic integers in the complex field $\mathbb{C}$ is a ring which we denote by $A$. Therefore $A \cap \mathbb{K}=O_{\Delta}$ is the ring of integers of the quadratic field $\mathbb{K}$ of discriminant $\Delta$. Set $w_{\Delta}=\frac{r-1+\sqrt{D}}{r}$ for $r$ defined in (1.3). Then $w_{\Delta}$ is called principal surd. We restate the ring of integers of $\mathbb{K}$ as $O_{\Delta}=\left[1, w_{\Delta}\right]=\mathbb{Z}\left[w_{\Delta}\right]$. In this case $\left\{1, w_{\Delta}\right\}$ is called an integral basis for $\mathbb{K}$. if

$I=\left[a, b+c w_{\Delta}\right]$ is a non-zero (quadratic) ideal of $O_{\Delta}$ if and only

$$
c|b, c| a \text { and } a c \mid N\left(b+c w_{\Delta}\right) .
$$

Furthermore for a given ideal $I$ the integers $a$ and $c$ are unique and $a$ is the least positive rational integer in $I$ which we will denote as $L(I)$. The norm of an ideal $I$ is defined as $N(I)=|a c|$. If $I$ is an ideal of $O_{\Delta}$ with $L(I)=N(I)$, i.e., $c=1$, then $I$ is called primitive which means that $I$ has no rational integer factors other than \pm 1 . Every primitive ideal can be uniquely given by $I=\left[a, b+w_{\Delta}\right]$. The conjugate of an ideal $I=\left[a, b+c w_{\Delta}\right]$ is defined as $\bar{I}=\left[a, \overline{b+c w_{\Delta}}\right]$. If $I=\bar{I}$, then $I$ is called ambiguous (see also [4], [2] and [3]).

Let $\delta$ denotes a real quadratic irrational integer with trace $t=\delta+\bar{\delta}$ and norm $n=\delta \bar{\delta}$. Thus $\bar{\delta}$ denotes its algebraic conjugate. Evidently given a real quadratic irrational $\gamma \in \mathbb{Q}(\delta)$, there are rational integers $P$ and $Q$ such that $\gamma=\frac{P+\delta}{Q}$ with $Q \mid(\delta+P)(\bar{\delta}+P)$. Hence for each $\gamma=\frac{P+\delta}{Q}$ there is a corresponding $\mathbb{Z}$-module $I_{\gamma}=[Q, P+\delta]$. In fact this module is an ideal by (1.4).

Two real numbers $\alpha$ and $\beta$ are said to be equivalent if there exists a $g=\left(\begin{array}{ll}r & s \\ t & u\end{array}\right) \in \bar{\Gamma}$ such that $g \alpha=\beta$, that is

$$
\frac{r \alpha+s}{t \alpha+u}=\beta .
$$

Given any quadratic irrational $\gamma=\frac{P+\delta}{Q}$, there exists an indefinite quadratic form

$$
\begin{aligned}
F_{\gamma}(x, y) & =Q(x-\delta y)(x-\bar{\delta} y) \\
& =Q x^{2}-(t+2 P) x y+\left(\frac{n+P t+P^{2}}{Q}\right) y^{2}
\end{aligned}
$$

of discriminant $\Delta=t^{2}-4 n$. Hence one associates with $\gamma$ an indefinite quadratic form $F_{\gamma}$ defined as above. Therefore if $\delta=\sqrt{D}$, then $t=0$ and $n=-D$. So $\Delta=4 D$, and if $\delta=\frac{1+\sqrt{D}}{2}$, then $t=1$ and 
$n=\frac{1-D}{4}$. So $\Delta=D$. The connection among $\gamma, I_{\gamma}$ and $F_{\gamma}$ is given by the following diagram:

$$
\begin{gathered}
\gamma=\frac{P+\delta}{Q} \\
F_{\gamma}(x, y)=Q(\stackrel{x}{\downarrow}-\delta y)(x-\bar{\delta} y)
\end{gathered}
$$

The opposite of $F_{\gamma}$ defined in (1.6) is

$$
\bar{F}_{\gamma}(x, y)=Q x^{2}+(t+2 P) x y+\left(\frac{n+P t+P^{2}}{Q}\right) y^{2}
$$

of discriminant $\Delta$.

We know that a quadratic form $F$ is said to be ambiguous if it is improperly equivalent to itself. Of course the surprising equivalence must interchange the numbers $\gamma=\frac{\delta+P}{Q}$ and its conjugate $\bar{\gamma}=\frac{\bar{\delta}+P}{Q}$. Thus if all is well the form $F_{\gamma}$ is ambiguous iff the number $\gamma$ is equivalent to its conjugate $\bar{\gamma}$. Therefore one sees that an ideal $I_{\gamma}$ is ambiguous if it is equal to its conjugate $\bar{I}_{\gamma}$. Hence the ideal $I_{\gamma}$ is ambiguous iff it contains both $\frac{\delta+P}{Q}$ and $\frac{\bar{\delta}+P}{Q}$ that is so iff

$$
\frac{\delta+P}{Q}+\frac{\bar{\delta}+P}{Q}=\frac{t+2 P}{Q} \in \mathbb{Z} .
$$

Therefore the condition $Q \mid(t+2 P)$ is the condition for a form $F_{\gamma}$ to be properly equivalent to its opposite $\bar{F}_{\gamma}$.

\section{Quadratic Irrationals, Quadratic Ideals And INDEFINITE QUADRATIC FORMS}

In this section we obtain some properties of quadratic irrationals $\gamma=$ $\frac{\delta+P}{Q}$, quadratic ideals $I_{\gamma}=[Q, \delta+P]$ and indefinite quadratic forms $F_{\gamma}(x, y)=Q x^{2}-(t+2 P) x y+\left(\frac{n+t P+P^{2}}{Q}\right) y^{2}$ which are obtained from $\gamma$. We consider the problem in two cases: $\delta=\sqrt{D}$ and $\delta=$ $\frac{1+\sqrt{D}}{2}$ for a positive non-square integer $D$.

First let assume that $\delta=\sqrt{D}$ and $Q=1$. Then $t=0$ and $n=-D$. Set $P=\frac{-p}{2}$ for prime $p$ such that $p \equiv 1,3(\bmod 4)$. Then

$$
\gamma_{1}=\frac{\delta+P}{Q}=\frac{\sqrt{D}+\frac{-p}{2}}{1}=\sqrt{D}-\frac{p}{2}
$$


and hence

$$
\begin{aligned}
I_{\gamma_{1}} & =\left[1, \sqrt{D}-\frac{p}{2}\right] \\
F_{\gamma_{1}}(x, y) & =x^{2}+p x y+\left(\frac{p^{2}-4 D}{4}\right) y^{2} .
\end{aligned}
$$

Now we can give some properties of $\gamma_{1}, I_{\gamma_{1}}$ and $F_{\gamma_{1}}$ by the following theorems.

Theorem 2.1. $\gamma_{1}$ is equivalent to its conjugate $\bar{\gamma}_{1}$ for every prime $p \equiv 1,3(\bmod 4)$.

Proof. Recall that $\gamma_{1}=\sqrt{D}-\frac{p}{2}$. Then the conjugate of $\gamma_{1}$ is $\bar{\gamma}_{1}=$ $-\sqrt{D}-\frac{p}{2}$. A straightforward calculations shows that

$$
g \bar{\gamma}_{1}=\frac{-1\left(-\sqrt{D}-\frac{p}{2}\right)+(-p)}{0\left(-\sqrt{D}-\frac{p}{2}\right)+1}=\frac{\sqrt{D}-\frac{p}{2}}{1}=\gamma_{1}
$$

for $g=\left(\begin{array}{ll}-1 & -p \\ 0 & 1\end{array}\right) \in \bar{\Gamma}$. Therefore by definition $\gamma_{1}$ is equivalent to its conjugate $\bar{\gamma}_{1}$.

Theorem 2.2. $I_{\gamma_{1}}$ is ambiguous for every prime $p \equiv 1,3(\bmod 4)$.

Proof. We know that an ideal $I_{\gamma}$ is ambiguous if it is equal to its conjugate $\bar{I}_{\gamma}$, or in other words iff $\frac{\delta+P}{Q}+\frac{\bar{\delta}+P}{Q}=\frac{t+2 P}{Q} \in \mathbb{Z}$. For $\delta=\sqrt{D}$ we have $t=0$, and hence $\frac{t+2 P}{Q}=\frac{2(-p / 2)}{1}=-p \in \mathbb{Z}$. Therefore $I_{\gamma_{1}}$ is ambiguous.

From Theorems 2.1 and 2.2 we can give the following result.

Corollary 2.3. $F_{\gamma_{1}}$ is properly equivalent to its opposite $\bar{F}_{\gamma_{1}}$ and is ambiguous for every prime $p \equiv 1,3(\bmod 4)$.

Proof. It is clear that $F_{\gamma_{1}}$ is properly equivalent to its opposite $\bar{F}_{\gamma_{1}}$ by (1.8) since $\frac{t+2 P}{Q}=-p \in \mathbb{Z}$. We know as above that an indefinite quadratic form $F_{\gamma}$ is ambiguous iff the quadratic irrational $\gamma$ is equivalent to its conjugate $\bar{\gamma}$. Therefore $F_{\gamma_{1}}$ is ambiguous since $\gamma_{1}$ is equivalent to its conjugate $\bar{\gamma}_{1}$ by Theorem 2.1 .

Now let $p \equiv 1,3(\bmod 4)$, i.e., $p=1+4 k$ or $p=3+4 k$ for a positive integer $k$, respectively. Then we have the following theorem. 
Theorem 2.4. If $F_{\gamma_{1}}$ is reduced, then

$$
D \in\left[4 k^{2}+2 k+1,4 k^{2}+6 k+2\right]-\left\{4 k^{2}+4 k+1\right\}
$$

for $p \equiv 1(\bmod 4)$, and if $F_{\gamma_{1}}$ is reduced, then

$$
D \in\left[4 k^{2}+6 k+3,4 k^{2}+10 k+6\right]-\left\{4 k^{2}+8 k+4\right\}
$$

for $p \equiv 3(\bmod 4)$. In both cases the number of these reduced forms is $p$.

Proof. Let $F_{\gamma_{1}}(x, y)=x^{2}+p x y+\left(\frac{p^{2}-4 D}{4}\right) y^{2}$ be reduced and let $p \equiv 1(\bmod 4)$. Then by definition, we have from $(1.2)$

$$
\begin{aligned}
|\sqrt{\Delta}-2| a|| & <b<\sqrt{\Delta} \\
& \Longleftrightarrow|\sqrt{4 D}-2| 1||<p<\sqrt{4 D} \Leftrightarrow 2|\sqrt{D}-1|<p<2 \sqrt{D} .
\end{aligned}
$$

Hence we get $D \geq 4 k^{2}+2 k+1$, since

$$
D>\frac{p^{2}}{4}=\frac{(1+4 k)^{2}}{4}=\frac{1+8 k+16 k^{2}}{4}=\frac{1}{4}+2 k+4 k^{2}
$$

and $D \leq 4 k^{2}+6 k+2$, since

$$
D<\frac{(p+2)^{2}}{4}=\frac{(3+4 k)^{2}}{4}=\frac{9+24 k+16 k^{2}}{4}=\frac{9}{4}+6 k+4 k^{2} .
$$

Consequently we have

$$
4 k^{2}+2 k+1 \leq D \leq 4 k^{2}+6 k+2
$$

Note that there exist $p+1$ indefinite reduced quadratic forms $F_{\gamma_{1}}$, since

$$
4 k^{2}+6 k+2-\left(4 k^{2}+2 k+1\right)+1=2+4 k=p+1 .
$$

But $D=4 k^{2}+4 k+1=\left(\frac{p+1}{2}\right)^{2} \in\left[4 k^{2}+2 k+1,4 k^{2}+6 k+2\right]$ is a square. So we have to omit it ( $D$ must be a square-free positive integer). Therefore there exist $p$ indefinite reduced quadratic forms $F_{\gamma_{1}}$ for $D \in\left[4 k^{2}+2 k+1,4 k^{2}+6 k+2\right]-\left\{4 k^{2}+4 k+1\right\}$.

Similarly, let $F_{\gamma_{1}}(x, y)=x^{2}+p x y+\left(\frac{p^{2}-4 D}{4}\right) y^{2}$ be reduced and let $p \equiv 3(\bmod 4)$. Then by definition, we have from $(1.2)$

$$
\begin{aligned}
|\sqrt{\Delta}-2| a|| & <b<\sqrt{\Delta} \\
& \Longleftrightarrow|\sqrt{4 D}-2| 1||<p<\sqrt{4 D} \Leftrightarrow 2|\sqrt{D}-1|<p<2 \sqrt{D} .
\end{aligned}
$$


Hence we get $D \geq 4 k^{2}+6 k+3$, since

$$
D>\frac{p^{2}}{4}=\frac{(3+4 k)^{2}}{4}=\frac{9+24 k+16 k^{2}}{4}=\frac{9}{4}+6 k+4 k^{2}
$$

and $D \leq 4 k^{2}+10 k+6$, since

$$
D<\frac{(p+2)^{2}}{4}=\frac{(5+4 k)^{2}}{4}=\frac{25+40 k+16 k^{2}}{4}=\frac{25}{4}+10 k+4 k^{2} .
$$

Consequently we have

$$
4 k^{2}+6 k+3 \leq D \leq 4 k^{2}+10 k+6 .
$$

Note that there exist $p+1$ indefinite reduced quadratic forms $F_{\gamma_{1}}$, since

$$
4 k^{2}+10 k+6-\left(4 k^{2}+6 k+3\right)+1=4 k+4=p+1 .
$$

But $D=4 k^{2}+8 k+4=\left(\frac{p+1}{2}\right)^{2} \in\left[4 k^{2}+6 k+3,4 k^{2}+10 k+6\right]$ is a square. So we have to omit it. Therefore there exist $p$ indefinite reduced quadratic forms $F_{\gamma_{1}}$ for $D \in\left[4 k^{2}+6 k+3,4 k^{2}+10 k+6\right]-$ $\left\{4 k^{2}+8 k+4\right\}$.

Example 2.1. Let $p=29 \equiv 1(\bmod 4)$. Then $\gamma_{1}=\sqrt{D}-\frac{29}{2}$ is equivalent to its conjugate $\bar{\gamma}_{1}$ for $g=\left(\begin{array}{ll}-1 & -29 \\ 0 & 1\end{array}\right) \in \bar{\Gamma}$. Also $I_{\gamma_{1}}=\left[1, \sqrt{D}-\frac{29}{2}\right]$ is ambiguous, and

$$
F_{\gamma_{1}}(x, y)=x^{2}+29 x y+\left(\frac{841-4 D}{4}\right) y^{2}
$$

is reduced for $D \in[211,240]$. But $D=225=15^{2} \in[211,240]$ is a square. Therefore $F_{\gamma_{1}}$ is reduced for $D \in[211,240]-\{225\}$. The number of these reduced forms is 29. Further $F_{\gamma_{1}}$ is properly equivalent to its opposite $\bar{F}_{\gamma_{1}}$ and is ambiguous.

Example 2.2. Let $p=43 \equiv 3(\bmod 4)$. Then $\gamma_{1}=\sqrt{D}-\frac{43}{2}$ is equivalent to its conjugate $\bar{\gamma}_{1}$ for $g=\left(\begin{array}{ll}-1 & -43 \\ 0 & 1\end{array}\right) \in \bar{\Gamma}$. Also $I_{\gamma_{1}}=\left[1, \sqrt{D}-\frac{43}{2}\right]$ is ambiguous, and

$$
F_{\gamma_{1}}(x, y)=x^{2}+43 x y+\left(\frac{1849-4 D}{4}\right) y^{2}
$$

is reduced for $D \in[421,462]$. But $D=441=21^{2} \in[421,462]$ is a square. Therefore $F_{\gamma_{1}}$ is reduced for $D \in[421,462]-\{441\}$. 
The number of these reduced forms is 43 . Further $F_{\gamma_{1}}$ is properly equivalent to its opposite $\bar{F}_{\gamma_{1}}$ and is ambiguous.

Now we consider the case $\delta=\frac{1+\sqrt{D}}{2}$ and $Q=1$. Then $t=1$ and $n=\frac{1-D}{4}$. Set $P=\frac{-(p+1)}{2}$ for prime $p$ such that $p \equiv 1,3(\bmod 4)$. Then

$$
\gamma_{2}=\frac{P+\delta}{Q}=\frac{\frac{-(p+1)}{2}+\frac{1+\sqrt{D}}{2}}{1}=\frac{-p+\sqrt{D}}{2}
$$

and hence

$$
\begin{aligned}
I_{\gamma_{2}} & =\left[1, \frac{-p+\sqrt{D}}{2}\right] \\
F_{\gamma_{2}}(x, y) & =x^{2}+p x y+\left(\frac{p^{2}-D}{4}\right) y^{2} .
\end{aligned}
$$

Theorem 2.5. $\gamma_{2}$ is equivalent to its conjugate $\bar{\gamma}_{2}$ for every prime $p \equiv 1,3(\bmod 4)$.

Proof. Recall that $\gamma_{2}=\frac{-p+\sqrt{D}}{2}$. The conjugate of $\gamma_{2}$ is $\bar{\gamma}_{2}=$ $\frac{-p-\sqrt{D}}{2}$. Applying (1.5), we get

$$
g \bar{\gamma}_{2}=\frac{-1\left(\frac{-p-\sqrt{D}}{2}\right)+(-p)}{0\left(\frac{-p-\sqrt{D}}{2}\right)+1}=\frac{-p+\sqrt{D}}{2}=\gamma_{2}
$$

for $g=\left(\begin{array}{ll}-1 & -p \\ 0 & 1\end{array}\right) \in \bar{\Gamma}$. Therefore by definition $\gamma_{2}$ is equivalent to its conjugate $\bar{\gamma}_{2}$.

Theorem 2.6. $I_{\gamma_{2}}$ is ambiguous for every prime $p \equiv 1,3(\bmod 4)$.

Proof. We know that an ideal $I_{\gamma}$ is ambiguous if it is equal to its conjugate $\bar{I}_{\gamma}$, or in other words iff $\frac{\delta+P}{Q}+\frac{\bar{\delta}+P}{Q}=\frac{t+2 P}{Q} \in \mathbb{Z}$. For $\delta=\frac{1+\sqrt{D}}{2}$ we have $t=1$, and hence $\frac{t+2 P}{Q}=\frac{1+2((-p-1) / 2)}{1}=-p \in \mathbb{Z}$. Therefore $I_{\gamma_{2}}$ is ambiguous.

From Theorems 2.5 and 2.6 we can give the following corollary.

Corollary 2.7. $F_{\gamma_{2}}$ is properly equivalent to its opposite $\bar{F}_{\gamma_{2}}$ and is ambiguous for every prime $p \equiv 1,3(\bmod 4)$. 
Proof. It is clear that $F_{\gamma_{2}}$ is properly equivalent to its opposite $\bar{F}_{\gamma_{2}}$ by (1.8) since $\frac{t+2 P}{Q}=-p \in \mathbb{Z}$, and is ambiguous since $\gamma_{2}$ is equivalent to its conjugate $\bar{\gamma}_{2}$ by Theorem 2.5 .

Theorem 2.8. If $F_{\gamma_{2}}$ is reduced, then

$$
D \in\left[16 k^{2}+8 k+2,16 k^{2}+24 k+8\right]-\left\{16 k^{2}+16 k+4\right\}
$$

for $p \equiv 1(\bmod 4)$, and if $F_{\gamma_{2}}$ is reduced, then

$$
D \in\left[16 k^{2}+24 k+10,16 k^{2}+40 k+24\right]-\left\{16 k^{2}+32 k+16\right\}
$$

for $p \equiv 3(\bmod 4)$. In both cases the number of these forms is $4 p+2$.

Proof. Let $F_{\gamma_{2}}(x, y)=x^{2}+p x y+\left(\frac{p^{2}-D}{4}\right) y^{2}$ be reduced and let $p \equiv 1(\bmod 4)$. Then by definition we have from $(1.2)$,

$|\sqrt{\Delta}-2| a||<b<\sqrt{\Delta}$

$$
\Longleftrightarrow|\sqrt{D}-2| 1||<p<\sqrt{D} \Leftrightarrow|\sqrt{D}-2|<p<\sqrt{D} .
$$

Hence we get $D \geq 16 k^{2}+8 k+2$, since

$$
D>p^{2}=(1+4 k)^{2}=1+8 k+16 k^{2}
$$

and $D \leq 16 k^{2}+24 k+8$, since

$$
D<(p+2)^{2}=(3+4 k)^{2}=9+24 k+16 k^{2} .
$$

Consequently we have

$$
16 k^{2}+8 k+2 \leq D \leq 16 k^{2}+24 k+8 .
$$

Note that there exist $4 p+3$ indefinite reduced quadratic forms $F_{\gamma_{2}}$, since

$16 k^{2}+24 k+8-\left(16 k^{2}+8 k+2\right)+1=16 k+7=4(1+4 k)+3=4 p+3$.

But $D=16 k^{2}+16 k+4=(p+1)^{2} \in\left[16 k^{2}+8 k+2,16 k^{2}+24 k+8\right]$ is a square. So we have to omit it. Therefore there exist $4 p+2$ indefinite reduced quadratic forms $F_{\gamma_{2}}$ for $D \in\left[16 k^{2}+8 k+2,16 k^{2}+24 k+\right.$ $8]-\left\{16 k^{2}+16 k+4\right\}$.

Similarly, let $F_{\gamma_{2}}(x, y)=x^{2}+p x y+\left(\frac{p^{2}-D}{4}\right) y^{2}$ be reduced and let $p \equiv 3(\bmod 4)$. Then by definition we have from $(1.2)$,

$$
\begin{aligned}
|\sqrt{\Delta}-2| a|| & <b<\sqrt{\Delta} \\
& \Longleftrightarrow|\sqrt{D}-2| 1||<p<\sqrt{D} \Leftrightarrow|\sqrt{D}-2|<p<\sqrt{D} .
\end{aligned}
$$


Hence we get $D \geq 16 k^{2}+24 k+10$, since

$$
D>p^{2}=(3+4 k)^{2}=9+24 k+16 k^{2}
$$

and $D \leq 16 k^{2}+40 k+24$, since

$$
D<(p+2)^{2}=(5+4 k)^{2}=25+40 k+16 k^{2} .
$$

Consequently, we have

$$
16 k^{2}+24 k+10 \leq D \leq 16 k^{2}+40 k+24 .
$$

Note that there exist $4 p+3$ indefinite reduced quadratic forms $F_{\gamma_{2}}$, since

$$
\begin{aligned}
16 k^{2}+40 k+24 & -\left(16 k^{2}+24 k+10\right)+1 \\
& =16 k+15=4(3+4 k)+3=4 p+3 .
\end{aligned}
$$

But $D=16 k^{2}+32 k+16=(p+1)^{2} \in\left[16 k^{2}+24 k+10,16 k^{2}+\right.$ $40 k+24]$ is a square. So we have to omit it. Therefore there exist $4 p+2$ indefinite reduced quadratic forms $F_{\gamma_{2}}$ for $D \in\left[16 k^{2}+24 k+\right.$ $\left.10,16 k^{2}+40 k+24\right]-\left\{16 k^{2}+32 k+16\right\}$.

Example 2.3. Let $p=73 \equiv 1(\bmod 4)$. Then $\gamma_{2}=\frac{-73+\sqrt{D}}{2}$ is equivalent to its conjugate $\bar{\gamma}_{2}$ for $g=\left(\begin{array}{ll}-1 & -73 \\ 0 & 1\end{array}\right) \in \bar{\Gamma}$. Also $I_{\gamma_{2}}=\left[1, \frac{-73+\sqrt{D}}{2}\right]$ is ambiguous, and

$$
F_{\gamma_{2}}(x, y)=x^{2}+73 x y+\left(\frac{5329-D}{4}\right) y^{2}
$$

is reduced for $D \in[5330,5624]$. But $D=5476=74^{2} \in[5330,5624]$ is a square. Therefore $F_{\gamma_{2}}$ is reduced for $D \in[5330,5624]-\{5476\}$. The number of these reduced forms is 294. Further $F_{\gamma_{2}}$ is properly equivalent to its opposite $\bar{F}_{\gamma_{2}}$ and is ambiguous.

Example 2.4. Let $p=83 \equiv 3(\bmod 4)$. Then $\gamma_{2}=\frac{-83+\sqrt{D}}{2}$ is equivalent to its conjugate $\bar{\gamma}_{2}$ for $g=\left(\begin{array}{ll}-1 & -83 \\ 0 & 1\end{array}\right) \in \bar{\Gamma}$. Also $I_{\gamma_{2}}=\left[1, \frac{-83+\sqrt{D}}{2}\right]$ is ambiguous, and

$$
F_{\gamma_{2}}(x, y)=x^{2}+83 x y+\left(\frac{6889-D}{4}\right) y^{2}
$$

is reduced for $D \in[6890,7224]$. But $D=7056=84^{2} \in[6890,7224]$ is a square. Therefore $F_{\gamma_{2}}$ is reduced for $D \in[6890,7224]-\{7056\}$. 
The number of these reduced forms is 334. Further $F_{\gamma_{2}}$ is properly equivalent to its opposite $\bar{F}_{\gamma_{2}}$ and is ambiguous.

\title{
REFERENCES
}

[1] R. A. Mollin, Quadratics, CRS Press, Boca Raton, New York, London, Tokyo, 1996.

[2] R. A. Mollin, Jocabi symbols, ambiguous ideals, and continued fractions, Acta Arith. 85 (1998), 331-349.

[3] R. A. Mollin, A survey of Jacobi symbols, ideals, and continued fractions, Far East J. Math. Sci. 6 (1998), 355-368.

[4] R. A. Mollin, K. Cheng, Palindromy and ambiguous ideals revisited, J. Number Theory $\mathbf{7 4}$ (1999), 98-110.

[5] A. Tekcan, O. Bizim, The connection between quadratic forms and the extended modular group, Mathematica Bohemica 128(3) (2003), 225-236.

\author{
Ahmet Tekcan and Hacer Özden, \\ Department of Mathematics, \\ Faculty of Science, \\ Uludag University, \\ Görükle 16059, \\ Bursa, Turkey \\ tekcan@uludag.edu.tr; hozden@uludag.edu.tr
}

Received on 9 March 2006 and in revised form on 1 September 2006. 